IOS Press

\title{
Bioinspired fingertip for anthropomorphic robotic hands
}

\author{
Marco Controzzi ${ }^{1, *}$, Marco D’Alonzo ${ }^{1}$, Carlo Peccia, Calogero Maria Oddo, \\ Maria Chiara Carrozza and Christian Cipriani \\ The BioRobotics Institute, Scuola Superiore Sant'Anna, Pontedera (P I), Italy
}

\begin{abstract}
.
BACKGROUND: An artificial fingertip with mechanical features and appearance similar to the human fingertip could represent a significant step forward towards the development of the next generation artificial hands. However, so far, a fingertip showing a good trade-off among mechanical features, appearance and anthropomorphism, along with its 3D computational model, is still missing.

OBJECTIVE: To explore and develop an artificial fingertip demonstrating a mechanical response similar to the human fingertip, in order to improve the grasp stability of robotic hands.

METHODS: Taking inspiration from the multi-layered structure of the human finger, novel artificial fingertips, composed of a rigid core and covered by layers of polymeric materials with different degrees of stiffness and topped by a hard nail were developed. An accurate 3D finite element (FE) model was also developed in order to simulate and evaluate the internal mechanical behavior of the prototypes under external indentations. The mechanical response of the prototypes was assessed and compared with that of the human fingertip and the FE model results, under different experimental conditions. Finally, the artificial fingertips were integrated into an anthropomorphic robotic hand and evaluated in grip tests, in order to compare the grasp stability with respect to conventional stiff (metal) fingertips.

RESULTS: The developed prototypes demonstrated a response to compression tests similar to the human finger and the FE model showed a discrete accuracy (mean error 7\%). Finally, an increased ability (by 96\%) in stably holding objects during precision grips with respect to conventional stiff fingers was demonstrated.
\end{abstract}

CONCLUSION: Multi-layered biomimetic fingertips can improve grasp stability and cosmetic appearance of anthropomorphic robot hands.

Keywords: Fingertip, grasping, manipulation, finite element analysis

\section{Introduction}

The ability to precisely manipulate objects through fingers i.e. dexterity, is one of the most amazing features of the human hand. This feature besides being enabled by modality-specific mechanoreceptors which are integrated into a sophisticated sensorimotor sys-

\footnotetext{
${ }^{1}$ These authors equally contributed to this work.

${ }^{*}$ Corresponding author: Marco Controzzi, The BioRobotics Institute, Scuola Superiore Sant'Anna, I-56025, Pontedera (P I), Italy. E-mail: marco.controzzi@ @ssup.it.
}

tem, is also possible due to the mechanical properties and physical structure of the fingers/fingertips [14]. For this reason a fingertip with structure and mechanical features similar to the biological model would represent a significant step forward towards the development of the next generation artificial hands.

The human fingertip comprises two skin layers (the epidermis and the dermis layers), subcutaneous fat tissues, the bone and the nail. All of these present different mechanical features $[6,14]$, and thus the whole structure shows a non-linear and time-dependent degree of stiffness, if mechanically stimulated. In particular 
the stiffness of the finger pad increases non-linearly with the force and speed of the stimulus. This peculiar feature plays a fundamental role during manipulation, precision grasping of objects and tactile perception [23]. In fact, while grasping and manipulating objects the softness of the fingertip enhances its conformability (by increasing the contact area) thus improving grasp stability, reducing contact pressures and risks of damaging fragile objects. When heavy objects are hand held and high forces are engaged, the pulps are compressed towards the nails which limit the deformation of the pulps and provide the sufficient rigidity required to accomplish the task. The stiffness of the finger pad is a time-dependent characteristic; it can be modeled as a function of the present and past speed of the mechanical stimulus and shows high hysteresis when stressed with loading and unloading indentation cycles. This feature is actually important as it allows the finger pad to absorb the energy from accidental impacts that might be experienced during grasping and catching of objects.

Several works investigated the mechanics and the response to mechanical stimuli by the human finger pad. Pawluk and Howe [22] and Wu et al. [32] studied the in vivo dynamic response when the finger pad is mechanically stimulated along the normal direction. Nakazawa et al. [16] and Pataky et al. [21] examined the impedance characteristics of the human finger pad along the tangential direction. Serina et al. [25] and Jindrich et al. [12] characterized the pulp response to repeated compressive loads. All these works agreed on the fact that the behavior of the pulp under indentation is represented by a non-linear function.

Several types of artificial fingertips were developed in order to mimic the non-linear mechanical behavior of the human finger. This non-linearity, and in general, the mechanical features of the human finger (e.g. resistance to wear, resistance to load, size, etc) cannot be replicated artificially by a single layer structure. In fact this is the result of a combination of layers each with different mechanical characteristics [1, 6]. Hence, finger pads comprising multiple layers were developed in the past. In particular: fingertips with a rigid core and a homogeneous compliant layer [15], with a rigid core, a homogeneous layer and a hard nail [30] and with a rigid core with multiple layers $[1,5,7,8,26,28,29$, 31]. Other studies compared the mechanical features of artificial fingertips to the human model; Chorley et al. [5] and Han and Kawamura [7] compared the stiffness, Han et al. [8] and Shao et al. [26] compared the friction. All these prototypes were developed but without the anthropomorphism/shape of the fingertip, with the result that they looked more like cylinders than like human fingers. However, in order to accurately mimick the non linear characteristic of the human fingertip the morphology/shape do count: the shape of the finger pad affects the mechanical response since it defines the distribution of the loads within the structure and across different materials (when present) [25] and thus it is imperative to be considered in the design.

Another key aspect of this research area relates to the development of computational models capable of describing accurately the mechanical behavior of the fingertip. Such a model is necessary for understanding the mechanical phenomena internal to the fingertip and to computationally evaluate the influence of the different design parameters (such as the thickness of the layers, the number of layers, the material properties, the bone shape, etc.) on the mechanical properties. The finite element method (FEM) was exploited for this purpose due to its ability to simulate complex nonlinear structures [1, 4, 6, 26, 33]. Dandekar et al. [6] developed a 3D multi-layered FE model of the human and of the monkey fingertips and demonstrated that their model could predict the responses to indentations of complex object shapes of the slowly adapting type I (i.e. SA I) mechanoreceptors. Wu et al. [33] modeled the dynamic response of the human fingertip with a multi-layered two-dimensional FE model. Shao et al. [26] developed a multi-layered 2D FE model in order to simulate the mechanical behavior of an artificial fingertip. Berselli et al. [1] used a 2D FE model that provided an estimate of hardness and friction influence on the static compliance of the finger pad. Cabibihan et al. [4] developed a 3D multi-layered FE model with the aim of comparing the force-displacement characteristic of an artificial finger with the experimental curves of the human phalanges; the simulated models were used to investigate the influence of the topology and materials used on the mechanical properties.

However, although several advancements were presented in the area of artificial hand design [1, 5], a fingertip showing a good trade-off among mechanical characteristics, appearance and anthropomorphism, along with its 3D computational model is still missing.

This work presents a novel bioinspired fingertip, composed of a bone (i.e. a rigid plastic core) covered by two polymeric layers with different degrees of stiffness and topped by a hard nail. The polymeric materials chosen were experimentally characterized in order to 
model their mechanical behaviors and to develop an accurate FE model of the global structure of the artificial fingertip. Different fingertips were manufactured and their mechanical response was evaluated and compared to the human fingertip and to the FE model results, under different conditions. The results showed that the prototypes demonstrated a response to compression tests similar to the human finger and that the FE model was accurate (mean error $7 \%$ ). Finally the importance of having compliant, biomimetic fingers was demonstrated by means of grasping tests using a robotic hand equipped with the developed fingertips. The ability to firmly hold objects in precision grips when using the biomimetic fingertips was increased by $96 \%$ with respect to stiff fingertips. These results open up promising possibilities for the development of the next generation artificial hands.

\section{Materials and methods}

\subsection{The human fingertip}

The finger pad comprises of several layers of tissues that cover the distal phalanx (hard bony structure) and a nail (keratinized skin cells) (Fig. 1a). The outer epidermal layer (epidermis) is thin $(<1 \mathrm{~mm})$, flexible and hard. This layer provides a strong resistance to tear, stretch and wear and protects/surrounds a softer dermal layer (dermis) and a very soft inner core of subcutaneous fat tissue matrix. The latter is, by volume, $60-72 \%$ water and is primarily composed of elastic adipose cells enmeshed in a network of loose fibrous tissue. Hence, forces normal to the epidermis produce large deformations allowing the finger pad to conform to surface edges with the smallest level of detail. Dorsally to the bone the nail bed (i.e. a harder skin structure) secures the nail and the skin to the bone. The nail, which has evolved out of claws, is a relatively flat curved carapace to protect the finger pad, which is functional for fingertip precision prehension and for suppressing excessive deformations of the soft tissues, in the case of palmar prehension, thus increasing the friction coefficient [15].

\subsection{Morphological design of the artificial fingertips}

Two fingertip morphologies were adopted and designed (named prototype $\beta$ and $\gamma$ in Fig. 1b, c), both based on the human fingertip. Both morphologies comprised of a multi-layer structure with a rigid core (that mimicked the geometry of the bone), two soft layers (that mimicked the epidermis and subcutaneous fat tissue, respectively) and a rigid nail on the dorsum. In both cases, all the layers had ellipsoid-like sections with different radius based on the work by Shao and colleagues [26]. The distal bone was placed approximately $2 \mathrm{~mm}$ towards the dorsal part of the fingertip (towards the nail) with respect to the center of the sections of the surrounding layers. The fingertip was bent by $20^{\circ} \mathrm{deg}$ in order to mimic the typical distalinterphalangeal (DIP) joint angle during activities of daily living [9]. The finger pad of the $\beta$ prototype (pad $\beta$ ) was designed with a large flat surface in order to present a large contact area from the onset of the contact. The angle of the plane of the flat surface was $20^{\circ}$ deg with respect to the principal axis of the fingertip in the sagittal plane. Prototype $\gamma(\operatorname{pad} \gamma)$ had the same geometry of $\beta$ except for the pulp of the tip that was rounded so mimicking the human curvature. The latter was experimentally measured from 40 index fingers of 20 human subjects and the average curvature (calculated using the major and minor semi-axes of the elliptic sections in the sagittal and transversal planes) was scaled to the dimensions of the artificial fingertip.

\subsection{Selection of the materials}

Measurements of the mechanical response of human finger pads (ex-vivo and in-vivo experiments) are the major source of reference in the literature $[6,13]$ (elastic modulus of bone, dermis and epidermis around $10^{4}$, $10^{-1}$ and $10^{-2} \mathrm{MPa}$, respectively) and were used for guiding our work. For each layer we chose materials from those commercially available with an elastic modulus comparable to that of the biological layer. Our objective indeed was to develop a prototype using solid (rather than fluid) materials, in order to integrate therein tactile sensors, in future works. We manufactured the bone using polyurethane resin (Axson PX2017, Young's Modulus of $1650 \mathrm{MPa}$ ) by means of vacuum casting molding technique. The multilayer soft structure of the fingertip was developed using custom molds and cold casting techniques (process is graphically described in Fig. 2a). Soft layers were manufactured using different kinds of silicone rubber: the outer layer, which reproduced the epidermis and dermis layers, was a commercial hyper-elastic silicone rubber (DragonSkin ${ }^{\circledR}$ Shore Hardness A-10, 
a)

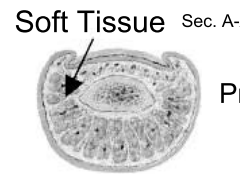

b)

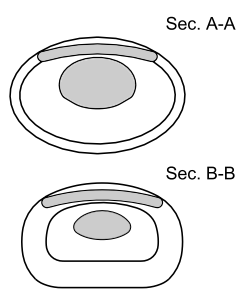

Proximal
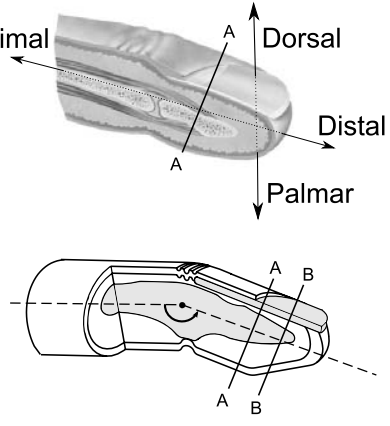

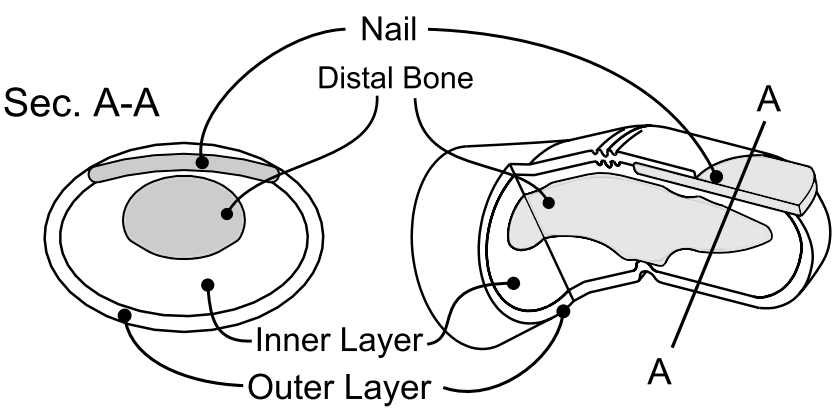

c)

Fig. 1. Structures of the human finger pad and of two artificial finger pads developed. (a) Transversal (Sec. A-A) and partial sagittal sections of the human finger pad. (b) Three sections of one of the artificial finger pads (i.e. pad $\beta$ ). The distal interphalangeal joint is missing but the finger is bent $\left(160^{\circ}\right.$ angle between the axis). (c) Two sections of a second finger pad (pad $\gamma$ ).

Table 1

Materials properties

\begin{tabular}{lcrrrrr}
\hline Material & Shore hardness & \multicolumn{3}{c}{ Elastic modulus (MPa) } & Ultimate strength (MPa) & Breaking elongation (\%) \\
\cline { 3 - 5 } & & \multicolumn{1}{c}{ At $-10 \%$} & \multicolumn{1}{c}{ At $-20 \%$} & At $+100 \%$ & & \\
\hline DragonSkin-10 & A-10 & $0.356 \pm 0.002$ & $0.421 \pm 0.005$ & $0.137 \pm 0.005$ & $0.812 \pm 0.072$ & $1623.7 \pm 93.9$ \\
EcoFlex 00-10 & $00-10$ & $0.05 \pm 0.000$ & $0.06 \pm 0.001$ & $0.0382 \pm 0.001$ & $0.730 \pm 0.116$ & $1237.6 \pm 106.1$ \\
EcoFlex 00-30 & $00-30$ & $0.0928 \pm 0.004$ & $0.109 \pm 0.008$ & $0.0477 \pm 0.001$ & $2.370 \pm 0.211$ & $1448.9 \pm 113.6$ \\
\hline
\end{tabular}

SmoothOn Inc.); for the inner layer, which reproduced the fat subcutaneous tissue, the two softest silicone rubbers commercially available were investigated: EcoFlex ${ }^{\circledR}$ Shore Hardness 00-10 (pads $\beta 1010$ and $\gamma$ 1010) and EcoFlex ${ }^{\circledR}$ Shore Hardness 00-30 (pads $\beta 3010$ and $\gamma$ 3010). Unfortunately it was not possible to find rubbers having a lower Shore Hardness. The characteristics of these materials, were measured by means of uniaxial compression and tensile tests, according to the ISO (International Organization for Standardization) procedures $[10,11]$. The results are shown in Table 1 and were used to implement the FEM.

\subsection{Finite elements model}

A multi-body three dimensional computational model was implemented in order to compute the mechanical response of the fingertips under the indentation of a flat rigid surface (indenter) in the normal direction (Fig. 3). The angle between the plane of the indenter and the distal bone axis was $20^{\circ} \mathrm{deg}$ in the sagittal plane (Fig. 3a, c). Materials were characterized using the 1st order Ogden model [18, 19] using the experimental data collected by means of uniaxial tests (parameters in Table 1). Geometries of the fingertips were imported in the Ansys ${ }^{\circledR} 14.0$ Workbench $^{\text {TM }}$ FEM environment (Ansys Inc., USA) directly from the CAD software $\left(\mathrm{Creo}^{\circledR} 2.0, \mathrm{PTC}^{\circledR}\right.$, USA). Several simplifications and hypotheses, reported hereafter, were made in order to reduce the computational cost and reach the convergence of the simulations.

- Only half of the CAD model (cut across the sagittal plane) was imported in the FEM environment and thus the geometrical symmetry was exploited (this was possible since only indentations along the normal direction were simulated).

- Since we could hypothesize that the strain would be localized on the finger pad, we chose not to include the proximal part of the fingertip in the model.

- In the interfaces between polymeric layers and rigid parts (bone and nail) the nodes of the polymeric materials were constrained to ground. This simplification was possible since the stiffness of the rigid parts was several order of magnitude higher than the polymeric materials and thus allowed the rigid part to be excluded from the simulation. 


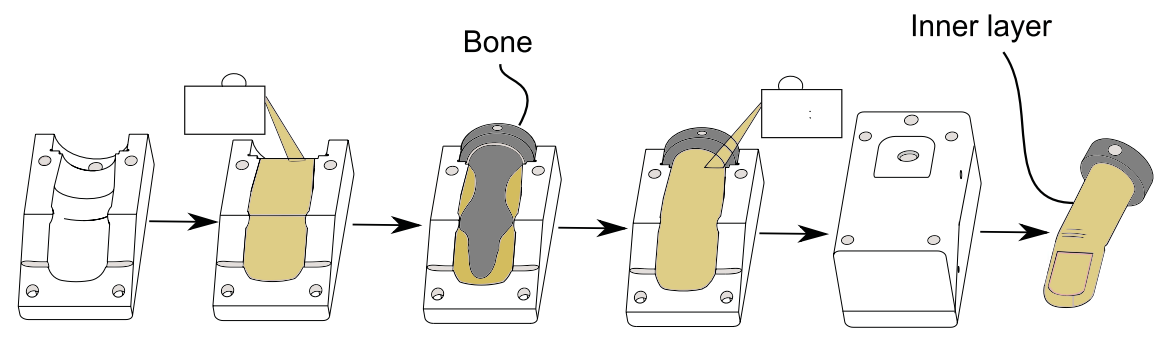

a)
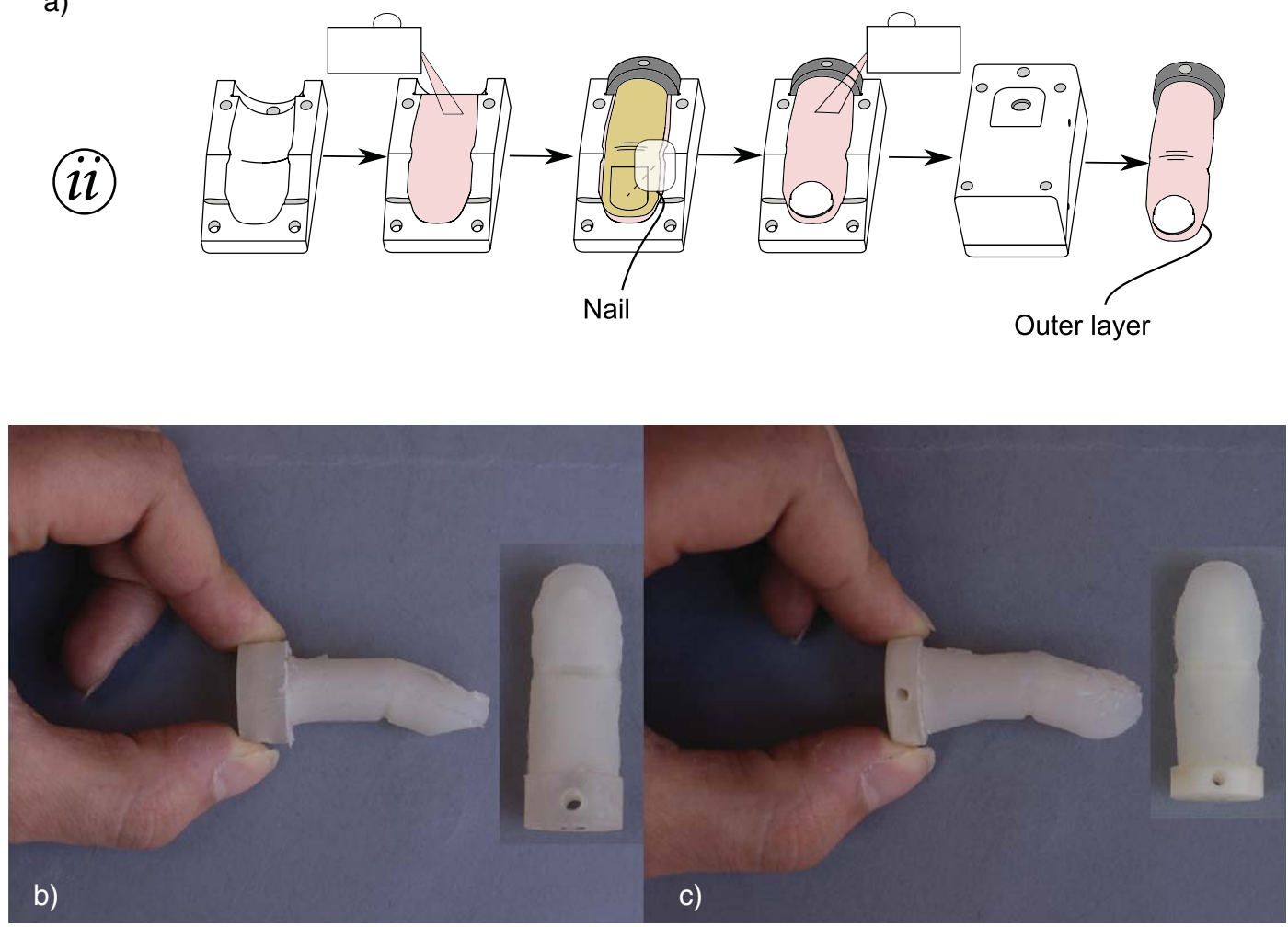

Fig. 2. Manufacturing process of the artificial fingertips. a) Schematic sequence of the cold molding, the process is subdivided into two-steps: i) the first mold is used for the fabrication of the inner layer on the artificial bone; (ii) the outer layer is realized using a second mold on the inner part of the finger. The nail is inserted at the final stage of the process. b) Pad $\beta$ prototype. c) Pad $\gamma$ compared with a human fingertip.

- Bonded-type contact behavior was chosen at the interface between the two soft layers since these were supposed to be polymerized and hence penetration, separation and sliding was not possible.

- The nodes at the interface between the pad and the indenter were considered in contact (and so included in the computation) only when they were closer than $0.2 \mathrm{~mm}$ (pinball region).

- The augmented Lagrange formulation was employed and the Normal Stiffness Factor (FKN) was chosen manually in order to achieve the optimal trade-off between convergence of the simulation and accuracy of the solution.

- The CAD model was meshed in two different steps. First the automatic mesh with fine size of the element was used for all the bodies; then, a spherical refinement centered on the nodes at the interfaces was manually added. As a result a total of $\sim 170000$ nodes and $\sim 8900$ elements was used in the FE models.

- Progressive displacements of the indenter were simulated in multiple steps allowing the software to add sub-steps in an iterative fashion in the case 

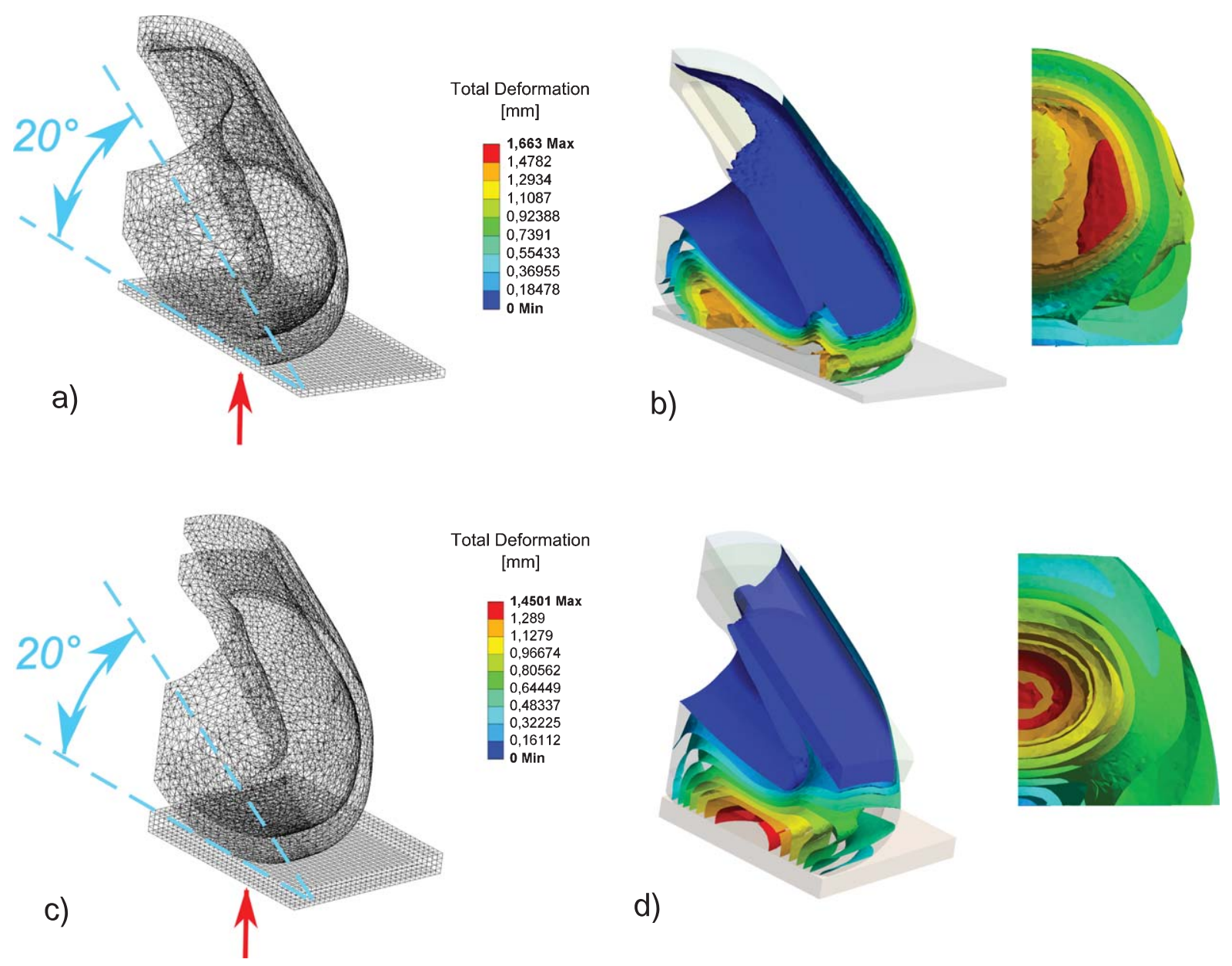

Fig. 3. Finite element models for pads $\beta(a, b)$ and $\gamma(c, d)$. Mesh discretization (sub-figures a, c) and predicted distributions of the displacements within the fingertips indented using a flat surface (sub-figures $b, d$ ) at the maximum indentation height.

the convergence failed. The simulations were conducted in static conditions.

The pictures in Fig. 3a, c show the mesh discretization for the FE models developed for pad- $\beta$ and $\gamma$ and the predicted distributions of the displacements within the fingertip indented using a flat surface Fig. 3b, d. The latter demonstrate that in pad- $\gamma$ the deformation is concentrated on the tip of the round pad; in pad- $\beta$ the deformation spreads out all over its flat part.

\subsection{Experimental indentation tests of the fingertips}

\subsubsection{Measurement of the stress-strain curves}

Following the manufacturing process described above (cf. Fig. 2) we developed several samples of the prototypes $\beta$ and $\gamma$ in order to characterize their mechanical behavior under normal loads and to evaluate the accuracy of the FE model. The tests consisted in pressing a flat aluminum plate (indenter) on the finger pads with controlled displacement (cf. Fig. 4) and in recording the generated reaction forces using a specific 2 degrees of freedom tactile stimulator [17]. To assess the similarity of the mechanical behavior of our fingertips to the human model, we performed identical experiments on the index fingers of human subjects $[N=8,6$ males, age $30 \pm 3$ (mean \pm standard deviation) years, thickness and width of the fingertips $13 \pm 2 \mathrm{~mm}$ and $16 \pm 2 \mathrm{~mm}$, respectively].

The tactile stimulator consisted of a linear electromagnetic voice coil actuator that allowed the indenter to press on the finger pad. The position of the voice 


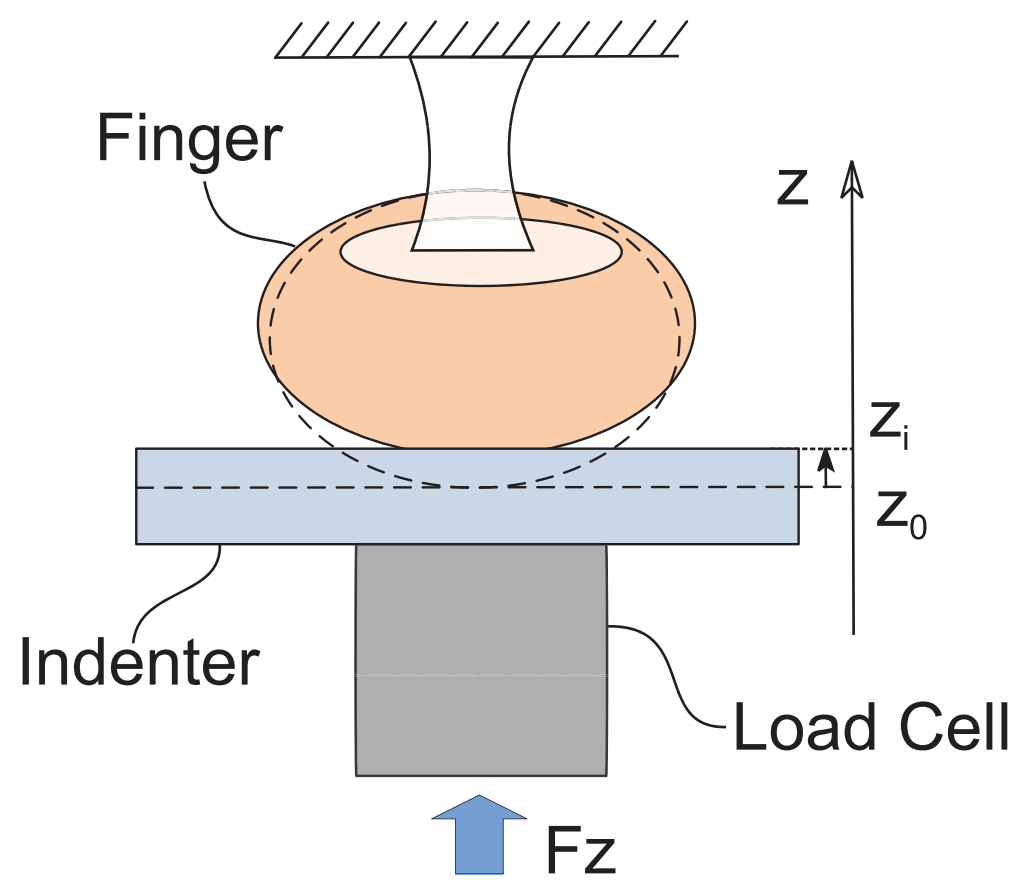

Fig. 4. Scheme for the indentation test: from the starting condition (indenter in $z_{0}$ ) the indenter was moved in the contact direction ( $\mathrm{z}$ ); the indenter position $\left(\mathrm{z}_{\mathrm{i}}\right)$ and the force (with a particular interest on the normal component $\mathrm{F}_{\mathrm{z}}$ ) generated by the deformation of the pad were recorded during the movement.

coil was measured by an optic sensor (Tonic T101115A, Renishaw plc., UK, micro-meter resolution). The contact force was measured by means of a sixaxis force/torque transducer (nano43, ATI Ltd., USA, milli-Newton resolution) placed above the voice coil actuator and topped by the indenter. Position control algorithms ran on a dedicated electronic board (DE2, Altera, USA) which communicated with an external PC over a serial bus. The data sampling frequency was $100 \mathrm{~Hz}$. A graphical user interface allowed the experimenter to run the experiment and to record data for offline analysis. The tactile stimulator was rigidly secured to ground and, during the experiments, the finger nails were glued onto a plastic support hinged to the platform (Fig. 5).

The maximum indentation level was set to $1.5 \mathrm{~mm}$. In the case of pad $\beta$ the tests were mainly aimed to compare the experimental results to the FE model. Hence the contact angle was $20^{\circ}$ deg (as in the FE model) and the indentation speed was $0.1 \mathrm{~mm} / \mathrm{s}$, i.e. quasi-static. In the case of pad $\gamma$ and of the human fingers two different contact angles $\left(20^{\circ}\right.$ and $30^{\circ}$ $\mathrm{deg})$ and three different indentation speeds $(0.1,0.5$ and $2.0 \mathrm{~mm} / \mathrm{s}$ ) were evaluated in order to perform a
Table 2

Experimental conditions for the indentation test

\begin{tabular}{lccc}
\hline Finger pad & \# of samples & $\begin{array}{c}\text { Contact angles } \\
{\left[{ }^{\circ} \mathrm{deg}\right]}\end{array}$ & $\begin{array}{c}\text { Indentation } \\
\text { speeds }(\mathrm{mm} / \mathrm{s})\end{array}$ \\
\hline$\beta 3010$ & 1 & 20 & 0.1 \\
$\beta 1010$ & 1 & 20 & 0.1 \\
$\gamma 3010$ & 3 & 20,30 & $0.1,0.5,2.0$ \\
$\gamma 1010$ & 3 & 20,30 & $0.1,0.5,2.0$ \\
Human & 8 & 20,30 & $0.1,0.5,2.0$ \\
\hline
\end{tabular}

comprehensive comparison between the artificial and the human finger pads. Each pad (artificial and human) was tested three times for each velocity/contact angle combination, for a total of 78 trials/recordings. Table 2 summarizes the conditions of the performed tests.

\subsubsection{Measurement of the contact area}

The contact area effectively established between the finger pad and a flat surface (angle $30^{\circ} \mathrm{deg}$ ) was measured for pads $\gamma 1010, \gamma 3010$ and the human index fingers $(N=8)$. To this aim the finger pads were dipped in ink and indented at different displacement heights (from 500 to $2500 \mu \mathrm{m}$ with $250 \mu \mathrm{m}$ increments) using the tactile platform topped by graph paper 


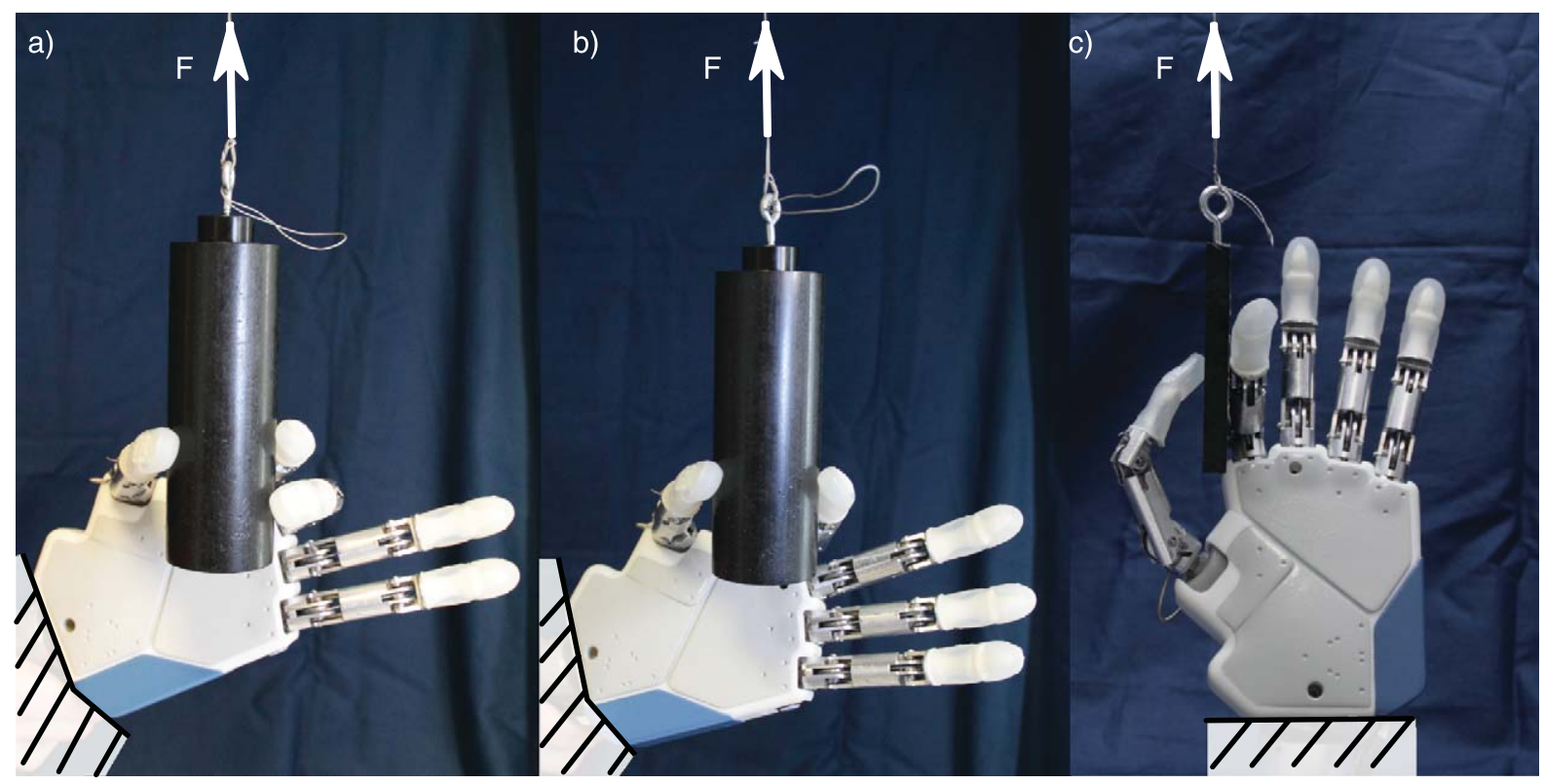

Fig. 5. Experimental assessment of the biomimetic fingertips on the robot hand. The plastic objects were pulled off the hand along the direction of the arrows. a) Tri-digital (thumb, index, middle) grip. b) Bi-digital grip (thumb, index). c) Lateral grip.

sheets. The speed of the indenter was to $0.1 \mathrm{~mm} / \mathrm{s}$. The fingerprints stamped on the graph paper sheets were digitized and the images were processed in MATLAB ${ }^{\circledR}$ (The MathWorks ${ }^{\circledR}$ Inc, USA) using a specific toolbox (Image Processing Toolbox ${ }^{\mathrm{TM}}$ ) in order to extract contact areas [20].

\subsection{Grip ability test with robotic hand}

The softness of the fingertip improves grasp stability in precision and lateral grips, i.e. in those grasps where the fingertip plays an essential role [27]. For this reason we evaluated the ability to firmly hold objects in precision and lateral grips using the new bioinspired fingertips $(\gamma$ 1010) integrated into a robot hand (IH2 Azzurra - Prensilia Srl, Italy). The maximum load force that the hand could hold in precision and lateral grasps was measured using the following setup. Plastic objects (Delrin ${ }^{\circledR}$, DuPont ${ }^{\mathrm{TM}}$, USA) with different size were grasped by the hand using automatic force-controlled grasps memorized within the hand. Once the grasp was achieved the hand was switchedoff and the object was pulled off at constant low speed $(0.1 \mathrm{~mm} / \mathrm{s})$ using a uniaxial testing machine (Instron ${ }^{\circledR}$, USA) so that the principal axis of the object was along the pulling direction of the testing machine and the object could slip through the hand (cf. Fig. 5). The hand was switched-off in order to measure only the effects of the fingertips (rather than those due to the controller of the hand). The minimum force required to pull off the object (pulling force) was measured by a data acquisition board. The procedure was repeated three times for each grip. The result were compared to those achieved when using stiff fingers under the same experimental conditions. Notably, the stiff fingers were covered by silicon tubes (thickness: $1 \mathrm{~mm}$ ) which had essentially the same friction coefficient with the environment of our fingertips $(2.2 \pm 0.2$ and $2.1 \pm 0.3$ for silicon tubes and DragonSkin ${ }^{\circledR}$, respectively). In this way we could compare fingertips with similar friction but different conformability.

\section{Results}

\subsection{Finite elements model}

The results from the FE model and the experimental indentation tests are shown for comparison in Fig. 6. As expected the computed curves showed a non-linear degree of stiffness with respect to the indentation (a larger indentation gave rise to larger degree of stiffness). Although we had incomplete information concerning the constitutive equations of the materials and we simplified the FE model the 
simulations showed results close to the experimental ones. In particular for pad $\beta 3010$ the model predicted the experimental curves with an error of $11 \pm 8 \%$ (mean \pm standard deviation) and a maximum error of $25 \%$. The error is slightly lower for pad $\beta 1010$ (mean $7 \pm 13 \%$, maximum error $23 \%$ ). With regards to pad $\gamma$ 3010, the model predicted the experimental curves with a mean error of $3 \pm 9 \%$ and a maximum error of $29 \%$. For pad $\gamma 1010$ the mean error was $8 \pm 5 \%$ and the maximum error was $15 \%$. Considering the $\gamma$ finger pad, the error between the FE model and the experimental curves was limited for displacements of the indenter up to $\sim 1000 \mu \mathrm{m}$, whereas above this value, it progressively increased; in fact the simple uniaxial modeling of the material poorly managed higher strain within the pad. For the same reason since the thickness of the internal layer was smaller in the $\beta$ pads than the $\gamma$ pads, the $\beta$ prototypes were subjected early to larger strain and the FE model was more prone to errors for displacements lower than in the $\gamma$ pads.

For sake of comparison, the stress-strain curves from the human fingers (indentation speed $0.1 \mathrm{~mm} / \mathrm{s}$ ) were also included in Fig. 7. The forces exerted were larger for pads $\beta$ than for pads $\gamma$ and importantly the forces engaged with pads $\beta$ were farther from the human curves than pads $\gamma$ (pad $\gamma$ was more similar to the human pad). Also for this reason, we chose to focus our analysis on pads $\gamma$.

\subsection{Experimental indentation tests of the fingertips}

\subsubsection{Measurement of the stress-strain curves}

The force-displacement curves as measured by the tactile stimulator on human and artificial pads $(\gamma 1010$ and $\gamma$ 3010) with different indentation speeds and angles are shown in Fig. 7. The indentation tests at different speeds demonstrated similar viscous trends between the two artificial pads and the human curves: the mechanical response was stiffer at higher indentation speed, as previously reported [7, 22]. Overall, the average relative difference between the stiffness of the artificial and human pads at maximum indentation $(1.5 \mathrm{~mm})$ and speed $(2.0 \mathrm{~mm} / \mathrm{s})$ were $90 \pm 30 \%$ for $\mathrm{pad}$ $\gamma 1010$ and $170 \pm 50 \%$ for pad $\gamma 3010$ with a indentation angle of $20^{\circ}$. These differences were smaller with an indentation angle of $30^{\circ}: 50 \pm 20 \%$ for pad $\gamma 1010$ and $100 \pm 30 \%$ for pad $\gamma 3010$. The observation of these results yields to the claim that a pad with an inner layer of a silicone rubber with a Shore Hardness below 00-10 would have mimicked the human behavior more closely than the developed pads. However (as recalled in the methods paragraph) the silicone rubber with Shore Hardness 00-10 was the softest commercially available. The average difference between curves at different angles was $12 \pm 1 \%$ for pad $\gamma 1010$, $11 \pm 1 \%$ for pad $\gamma 3010$ and $25 \pm 8 \%$ for the human pad. Overall, the pad showing the mechanical response closer to the human finger was $\gamma 1010$.

\subsubsection{Measurement of the contact area}

A representative fingerprint stamp series at different indentations is showed in Fig. 8a, whereas the graph in Fig. $8 \mathrm{~b}$ shows the experimental data at different indentations and linear interpolations. The contact area generated by the artificial pads was similar (average differences $13 \pm 3 \%$ for pad $\gamma 1010$ and $24 \pm 4 \%$ for pad $\gamma 3010)$ to the human data for indentations below 1000 $\mu \mathrm{m}$. For larger indentations the difference was larger but still below 25\% (20 $\pm 3 \%$ for pad $\gamma 1010$ and $24 \pm$ $3 \%$ for pad $\gamma 3010)$. In fact the linear coefficient of the linear fitting was larger for the human finger pad $(0.082$ $\mathrm{mm}^{2} / \mu \mathrm{m}$ vs. $0.063 \mathrm{~mm}^{2} / \mu \mathrm{m}$ and $0.060 \mathrm{~mm}^{2} / \mu \mathrm{m}$ for pads $\gamma 1010$ and $\gamma 3010$, respectively), similarly to previous literature [7]. Also for this test the finger pad $\gamma 1010$ showed to be the most biomimetic.

\subsection{Grip ability test with robotic hand}

Based on the results obtained in the different tests we chose to integrate pad $\gamma 1010$ in the robotic hand available (Fig. 5). Table 3 summarizes the pulling force (the static external disturbance that the hand can sustain before the grasp fails due to slippage) for precision and lateral grasps when using the hand with compliant or stiff fingers. As expected the pulling force was larger with the developed finger pads. The prehensile forms which most benefitted from the compliant fingers were the bi-digital (pulling force increased by $75.1 \%$ vs. the stiff fingers) and the tri-digital $(+177.1 \%)$. A reduced increment was seen for the lateral grasp $(+35.6 \%)$ probably due to the fact that only one finger pad was involved.

\section{Discussion}

The choice of a three layers structure was steered by the morphology of the biological human model, which presents a compliant behavior during grasps, resistance to wear and to external loads. In simple 

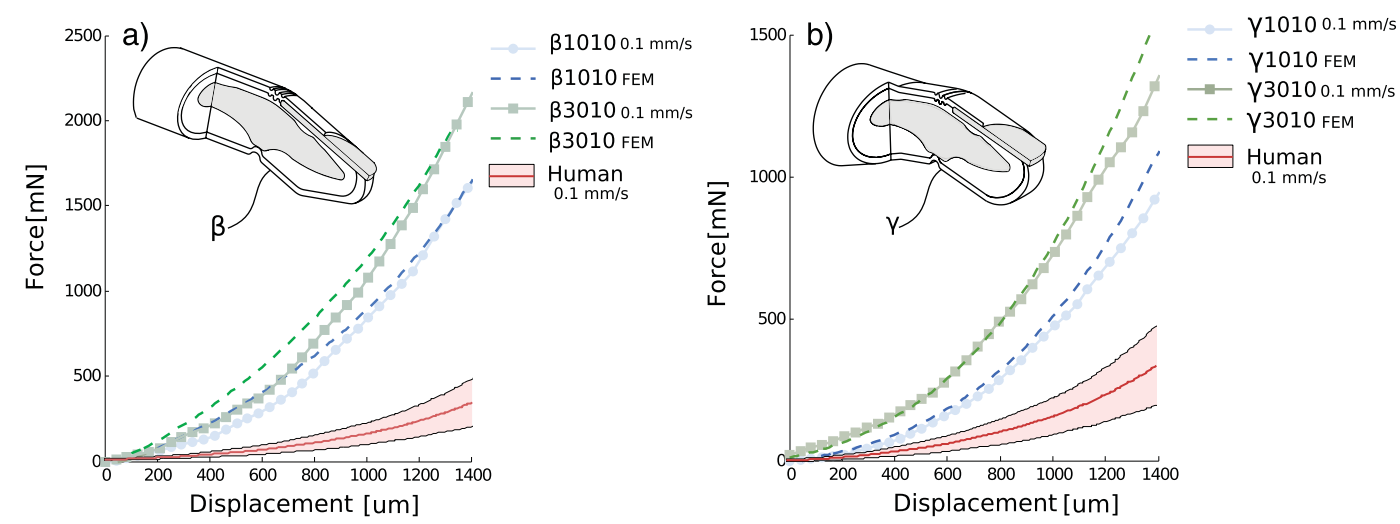

Fig. 6. Stiffness curves for pad $\beta$ and $\gamma$. Experimental results at low indentation speed $(0.1 \mathrm{~mm} / \mathrm{s})$, FEM results in static conditions for pad $\beta$ (a) and $\operatorname{pad} \gamma(\mathrm{b})$ and curves obtained from human measurements (filled areas include all subjects tested at $0.1 \mathrm{~mm} / \mathrm{s}$; solid line is the mean value). It is worth noting the different force scales in the two graphs.
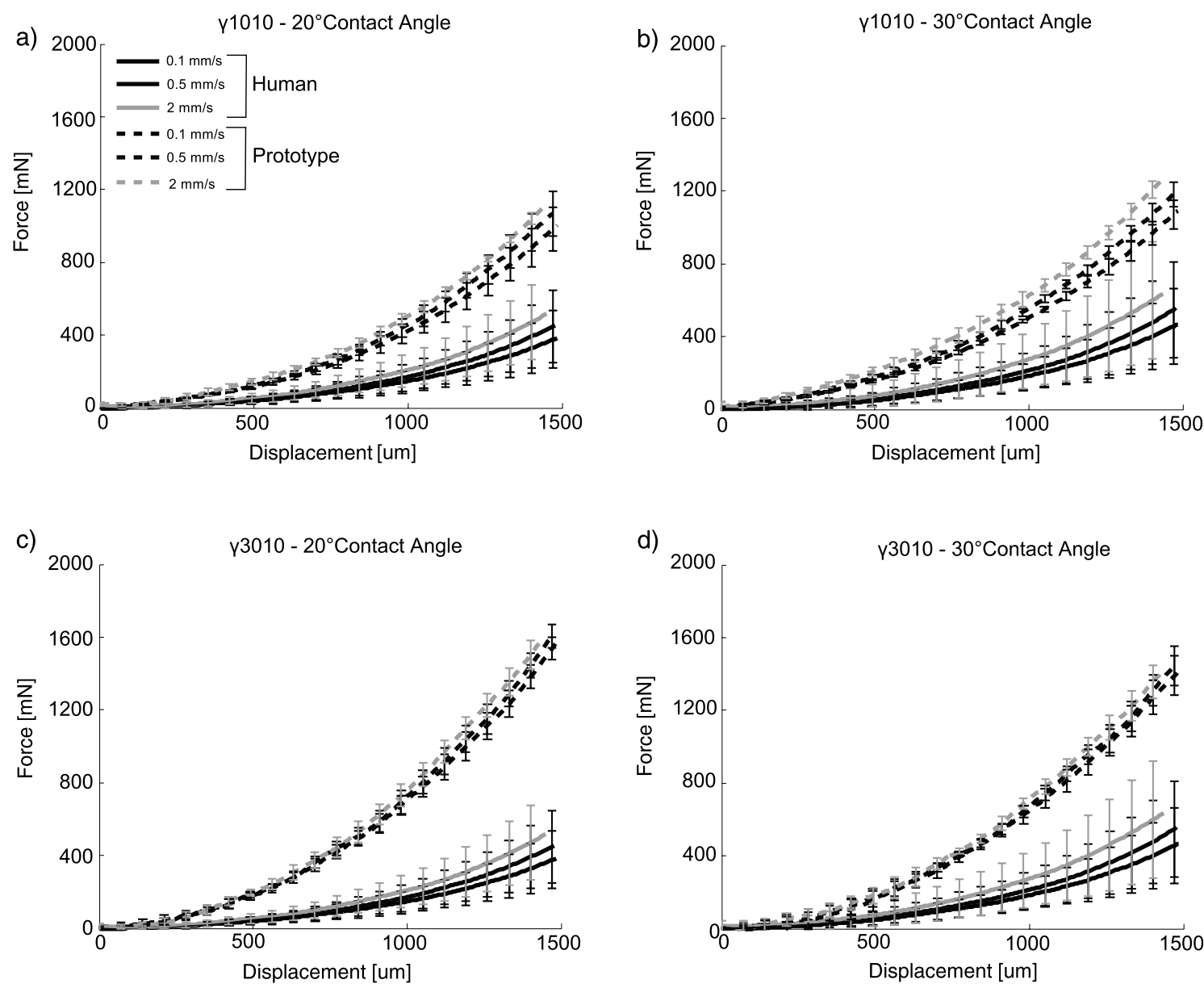

Fig. 7. Experimental stiffness curves at different velocities $(0.1,0.5,2 \mathrm{~mm} / \mathrm{sec})$ and contact angles $\left(20^{\circ}\right.$ and $\left.30^{\circ}\right)$. Error bars represent the standard deviation. 

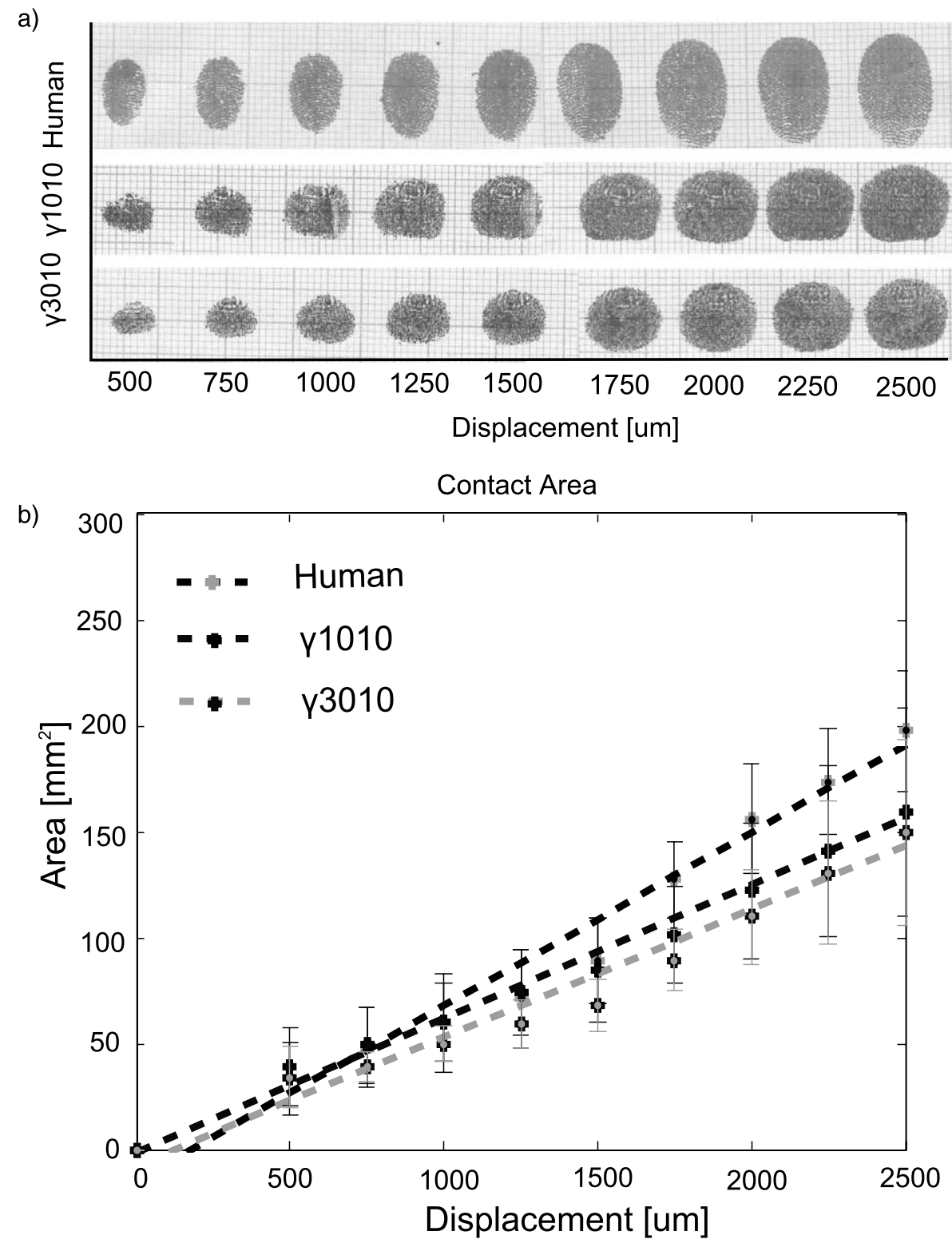

Fig. 8. Contact areas during touch. a) Fingerprints stamped on graph paper by one representative human pad and by pads $\gamma 1010$ and $\gamma 3010$ at different indentations. b) Contact areas at different indentations.

words, supported by the experience gained through this work, we can state that in an artificial pad the compliance is mainly due to the softness of the inner layer, the resistance to wear is due to the hard skin, while the bone prevents excessive torsions under external loads. However, as shown by our results (Fig. 7), even if we successfully replicated the human fingertip size, general structure and appearance, this was not enough to achieve a comparable stiffness. Our pads were stiffer than the human fingers. As mentioned above one could use softer materials for the inner layer, however, we could not find a commercial polymeric rubber with a Shore Hardness lower than the one used (00-10) [5]. The use of soft gel, foam or liquids exhibiting a lower Shore Hardness would probably increase the compliance of the finger pad. Nevertheless, artificial fingers that integrate these materials, present other practical problems; for example they would have to be hermet- 
Table 3

Grasping capabilities evaluation - force expressed in Newton ${ }^{\mathrm{a}}$

\begin{tabular}{|c|c|c|c|c|c|c|}
\hline \multirow[t]{2}{*}{ Diameter cylinder } & \multicolumn{2}{|c|}{ Tri-digital } & \multicolumn{2}{|c|}{ Bi-digital } & \multicolumn{2}{|c|}{ Lateral } \\
\hline & $\mathrm{S}$ & $\mathrm{C}$ & $\mathrm{S}$ & $\mathrm{C}$ & $\mathrm{S}$ & $\mathrm{C}$ \\
\hline $2 \mathrm{~mm}$ thin plate & - & - & - & - & $4.5 \pm 1.5$ & $6.1 \pm 1.1$ \\
\hline Cylinder Ø $12 \mathrm{~mm}$ & - & - & $1.2 \pm 0.8$ & $5.0 \pm 0.7$ & - & - \\
\hline Cylinder $\varnothing 41 \mathrm{~mm}$ & $3.4 \pm 0.8$ & $7.2 \pm 0.8$ & $3.2 \pm 1.5$ & $7.2 \pm 1.0$ & - & - \\
\hline Cylinder Ø $51 \mathrm{~mm}$ & $4.2 \pm 1.2$ & $8.7 \pm 0.5$ & $3.9 \pm 1.3$ & $7.4 \pm 0.8$ & - & - \\
\hline Cylinder Ø 71 mm & $6.9 \pm 0.6$ & $10.6 \pm 1.5$ & - & - & - & - \\
\hline Cylinder Ø 102 mm & $10.1 \pm 1.3$ & $12.5 \pm 2.0$ & - & - & - & - \\
\hline
\end{tabular}

${ }^{a}$ Data based on three measurements. $\mathrm{S}$ is for artificial hand equipped with stiff fingertips. $\mathrm{C}$ is for artificial hand equipped with compliant fingertips.

ically sealed and might need systems for monitoring the internal state (e.g. pressure, to avoid leakages) $[1,31,34]$. As a side effect these solutions would increase the complexity and hence the manufacturing costs. In our case the soft rubber will allow to integrate commercially available tactile sensors inside the finger pad (e.g. Force Sensing Resistors or strain gauges).

The different experimental and simulated results obtained with pads $\beta$ confirmed the importance of the shape of the fingertip in the mechanical response to indentation. The lower degree of stiffness exhibited by pad $\gamma$ is due to both the larger thickness of the inner layer (compared to pad $\beta$ ) and to the rounded tip which gradually conforms during the indentation, as shown by the contact area analysis (Fig. 8).

Although the artificial fingertips were stiffer than the biological ones, since the curvature of pad $\gamma$ was properly geometrically scaled, we were able to find comparable results in the measurements of the contact areas vs. the indentation displacement.

With regard to the FE model, in order to fully mechanically characterize a hyper-elastic material, several tests are required: i) uniaxial test, ii) biaxial test iii) shear test, and iv) volumetric test. We only used data from a uniaxial characterization (compression and tensile tests). Although this was a partial characterization, it yielded to limited errors (experimental data vs. simulations), in a small range of indentation. However, it is noteworthy that manipulation and precision grips usually involve small ranges of force and indentation [22]. Another limitation of the model is that it can describe only static loading conditions, however this information can be enough to provide rough estimates of force/deformation values when evaluating different materials and/or morphologies. Finally, it is worth to note that the FE simulations were made considering the nail fixed to ground and with an external pressure applied on the pad, in order to replicate the experi- mental conditions. This explains the absence of nail bed deformation in the FE results, which is instead found in response to pressure on human finger pads. The effect is easily visible in the change in color of the nail bed due to redistribution of blood in the capillaries of the nail bed, and was shown to be important for force encoding [3].

The objective of our 3D model was significantly different compared to the other one found in the literature [6]. In fact, Dandekar and colleagues developed a model of the human finger pad by merging available and newly measured experimental data from human and monkey finger pads [6]. Apart from this, in their model they assumed linear elasticity (linear stressstrain mechanical behavior), while in our work we considered the non-linear elastic behavior of the materials by means of a hyper-elastic model. Our work was more similar to that of Cabibihan et al. [4] which employed a 3D virtual model, in order to simulate the behavior of artificial pads to a mechanical stimulus. However, the goodness of their virtual 3D model was not compared with experimental measurements and they tested a quite limited set of materials / morphologies [i.e. one kind of silicone (Shore Hardness A 11) ad one kind of polyurethane (Shore Hardness A 40)].

In the human fingertip the nail is anchored to a specific structure, the nail-bed. This structure holds stably the nail. In our prototypes the nail was embedded between the skin and the soft inner layer, on top of the bone, in order to mimic the human morphology. However, this did not turn to be effectively anchored as its stability depended only on the strength of the connection between the two silicone rubber layers and the nail. This imperfect stability, although it did not influence our outcomes, could make the finger not robust enough for integration into a robotic hand. However this issue can be fixed, by attaching the nail to the rigid bone thus making it a unique rigid structure. 
Finally, it is noteworthy that with respect to the choice of a fixed angle of $20^{\circ} \mathrm{deg}$ for the distal joint, while this angle is less disadvantageous than others [9], loss of the ability to flex this joint could lead to problems in humans during reaching and grasping [24] or to unstable grasps with stiff robotic hands [2]. However, this lack should be mitigated by the presence of a multi-layered soft structure which improves conformability, as described in the literature [27] and shown in our tests with the robotic hand.

The design presented here offers a demonstration of how biologically inspired fingertips for robotic hands could gain some of the qualities that the human fingers have in terms of conformability and compliance.

\section{Conclusion}

The aim of this work was to develop an artificial fingertip inspired by the human fingertip that could be integrated in anthropomorphic robotic hands. Taking inspiration from the morphology of the human finger, we developed several artificial prototypes composed by a rigid inner bone, a thin rigid nail and two soft polymeric layers. For the latter we evaluated several materials and shapes in order to converge to the human mechanical response. The chosen morphology was inspired by the dimensions, proportions and structure of the human fingertip (i.e. the multi-layer structure). The prototypes were easy to manufacture (using relatively low cost techniques) and showed a mechanical behavior similar to the human one, as demonstrated by the experimental measures. In addition a 3D FE computational model was developed. The key outcome of the FE model is the opportunity to predict the behavior of the inner parts of the finger pads under external loads. The artificial fingertips were finally integrated onto a robotic hand and tested in grip tests, demonstrating their ability to conform on objects while grasping. We experimentally showed that artificial hands equipped with compliant fingers like the ones presented here could be more efficient in grasping. Future works will regard the integration of sensing elements in the bulk of the pad; the FE model will be crucial for understanding where to fix the sensors within the structure.

\section{Acknowledgments}

This work was supported in part by the European Commission under the COGLABORATION project
(EU-FP7-ICT-287888) and by the Italian Ministry of Education University and Research, under the FIRB2010 MY-HAND Project [RBFR10VCLD]. Authors would like to thank D. Camboni and A. Remazeilles for significant advices on the manuscript.

\section{References}

[1] G. Berselli, M. Piccinini, G. Palli and G. Vassura, Engineering design of fluid-filled soft covers for robotic contact interface: Guidelines, Nonlinear modeling, and experimental validation, IEEE Transaction on Robotics 27(3) (2011), 436-449.

[2] L. Birglen and C. Gosselin, Kinetostatic analysis of underactuated fingers, IEEE Transactions on Robotics and Automation 20(2) (2004), 211-221.

[3] I. Birznieks, V.G. Macefield, G. Westling and R.S. Johansson, Slowly adapting mechanoreceptors in the borders of the human fingernail encode fingertip forces, Journal of Neuroscience 29(29) (2009), 9370-9379. doi: 10.1523/JNEUROSCI.0143-09.2009.

[4] J.J. Cabibihan, R. Pradipta and S.S. Ge, Prosthetic finger phalanges with lifelike skin compliance for low-force social touching interactions, Journal of Neuroengineering and Rehabilitation 8(16) (2011) doi:10.1186/1743-0003-8-16

[5] C. Chorley, C. Melhuish, T. Pipe, J. Rossiter and G. Whiteley, A biologically inspired fingertip design for compliance and strenght, in Proceeding of Towards Autonomous Robotic Systems, (TAROS) (2008), 239-244.

[6] K. Dandekar, B. Raju, I. and M.A. Srinivasan, 3-D finite-element model of human and monkey fingertips to investigate the mechanics of tactile sense, Journal of Biomechanical Engineering-T ASME 125 (2004), 682-691.

[7] H.Y. Han and S. Kawamura, Analysis of stiffness of human fingertip and comparison with artificial fingers, in Systems, Man, and Cybernetics, 1999. IEEE SMC '99 Conference Proceedings 1999 IEEE International Conference on 2 (1999), 800-805.

[8] H.Y. Han, A. Shimada and S. Kawamura, Analysis of friction on human finger and design of artificial finger, in Robotics and Automation, 1996. Proceedings 1996 IEEE International Conference on 4 (1996), 3061-3066.

[9] M.C. Hume, H.Gellman, H. McKellop and R.H. Blumfield, Functional range of motion of the joints of the hand, Journal of hand surgery 15(2) (1990), 240-243.

[10] ISO 37:2005 (E) 2005 Rubber, vulcanized or thermoplastic-Determination of tensile stress-strain properties.

[11] ISO 7743:2008 (E) 2008 Rubber, vulcanized or thermoplastic-Determination of compression stress-strain properties.

[12] D.L. Jindrich, Y. Zhou, T. Becker and J. Dennerlein, Non-linear viscoelastic models predict fingertip pulp force-displacement characteristics during voluntary tapping, Journal of Biomechanics 36(4) (2003), 497-503.

[13] J.V. Kimberly and M.A. Srinivasan, Investigation of internal geometry and Mechanics of the human fingertip, in vivo, using Magnetic Resonance Image, Rle Technical Report No. 622 (1998).

[14] T. Maeno, K. Kobayashi and N. Yamazaki, Relationship between the structure of human finger tissue and the location of tactile receptors, JSME Int J 41 (1998), 94-100 
[15] K. Murakami and T. Hasegawa, Novel fingertip equipped with soft skin and hard nail for dexterous multi-fingered robotic manipulation in robotics and automation, 2003, Proceedings ICRA 'O3. IEEE International Conference on 1 (2003), $708-713$.

[16] N. Nakazawa, R. Ikeura and H. Inooka, Characteristics of human fingertips in the shearing direction, Biological Cybernetics 82(3) (2000), 207-214.

[17] C.M. Oddo, L. Beccai, N. Vitiello, H. Backlund Wasling, J. Wessberg and M.C. Carrozza, A mechatronic platform for human touch studies, Mechatronics 21(3) (2011), 604-613.

[18] R.W. Ogden, Large deformation isotropic elasticity-on the correlation of theory and experiment for incompressible rubberlike solids, Proceedings of the Royal Society of London, Series A, Mathematical and Physical Sciences 326(1567) (1972), 565-584.

[19] R.W. Ogden, G. Saccomandi and I. Sgura, Fitting hyperelastic models to experimental data, Computational Mechanics 34(6) (2004), 484-502.

[20] N. Otsu, A threshold selection method from gray-level histograms, IEEE Transactions on Systems, Man, and Cybernetics 9(1) (1979), 62-66.

[21] T.C. Pataky, M.L. Latash and M.L. Zatziosky, Viscoelastic response of the finger pad to incremental tangential displacements, Journal of Biomechanics 38 (2005), 1441-1449.

[22] D.T.V. Pawluk and R.D. Howe, Dynamic lumped element response of the human finger pad, Journal of Biomechanical Engineering 121(2) (1999), 178-183.

[23] R. Pfeifer, F. Iida and G. Gómez, Morphological computation for adaptive behavior and cognition, International Congress Series 1291 (2006), 22-29.

[24] M. Santello and J.F. Soechting, Gradual molding of the hand to object contours, Journal of Neurophysiology 79 (1998), 1307-1320.

[25] E. Serina, C. Mote and D. Rempelt, Force response of the fingertip pulp to repeated compression-effects of loading rate, loading angle and antropometry, Journal of Biomechanics 30(10) (1997), 1035-1040.

[26] F. Shao, T.H.C. Childs and B. Henson, Developing an artificial fingertip with human friction properties, Tribology International 42 (2009), 1575-1581.

[27] K.B. Shimoga and A.A. Goldenberg, Soft materials for robotic fingers, robotics and automation, 1992, Proceedings 1992 IEEE International Conference on 2 (1992), 1300-1305.

[28] Y. Tada, K. Hosoda, Y. Yamasaki and M. Asada, Sensing the texture of surfaces by anthropomorphic soft fingertips with multimodal sensors, in Intelligent Robots and Systems, 2003, (IROS 2003), Proceedings 2003 IEEE/RSJ International Conference on 1 (2003), 31-35.

[29] S. Takamuku, T. Iwase and K. Hosoda, Robust material discrimination by a soft anthropomorphic finger with tactile and termal sense, in Intelligent Robots and Systems, 2008 IROS 2008. IEEE/RSJ International Conference on (2008), 3977-3982.

[30] P. Tiezzi and G. Vassura, Experimental analysis of soft fingertip with internal rigid core, Advanced Robotics, 2005. ICAR '05. Proceedings, 12th International Conference on (2005), 109-114.

[31] N. Wettels, V.J. Santos, R. Johansson and G. Loeb, Biomimetic tactile sensor array, Advanced Robotics 22(8) (2008), 829-849.

[32] J.Z. Wu, R.G. Dong, W. Smutz and S. Rakheja, Dynamic interaction between a finger pad and a flat surface: Experiments and analysis, Medical Engineering \& Phisics 25(5) (2003), 397-406.

[33] J.Z. Wu, D.E. Welcome, K. Krajinak and R.G. Dong, Finite element analysis of penetrations of shear and normal vibrations into the soft tissues in a fingertip, Medical Engineering \& Physics 29 (2007), 718-727.

[34] T. Yamamoto, N. Wettels, J.A. Fishel, C.H. Lin and G.E. Loeb, BioTac, Journal of the Robotics Society of Japan 30(5) (2012), 496-498. 

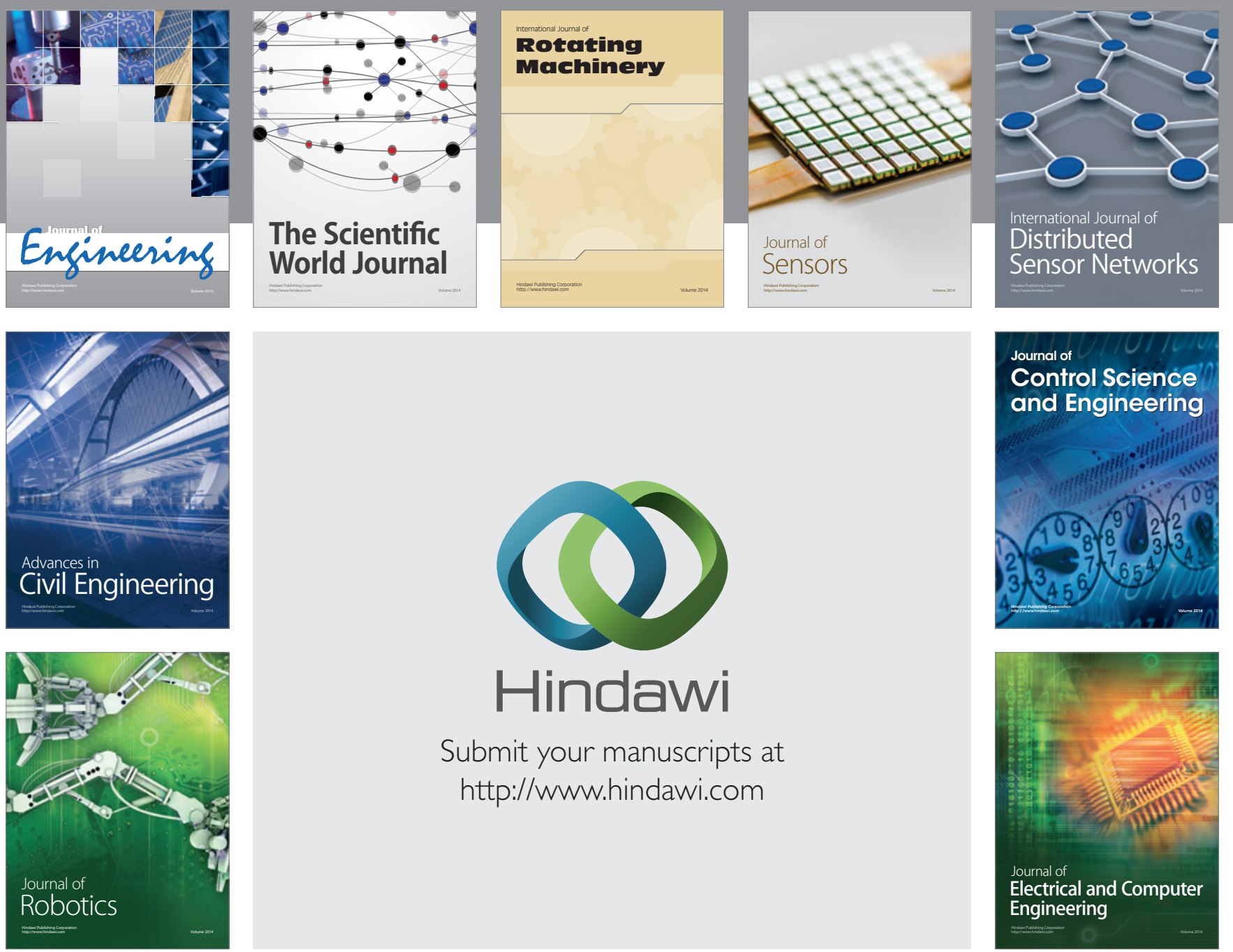

Submit your manuscripts at

http://www.hindawi.com
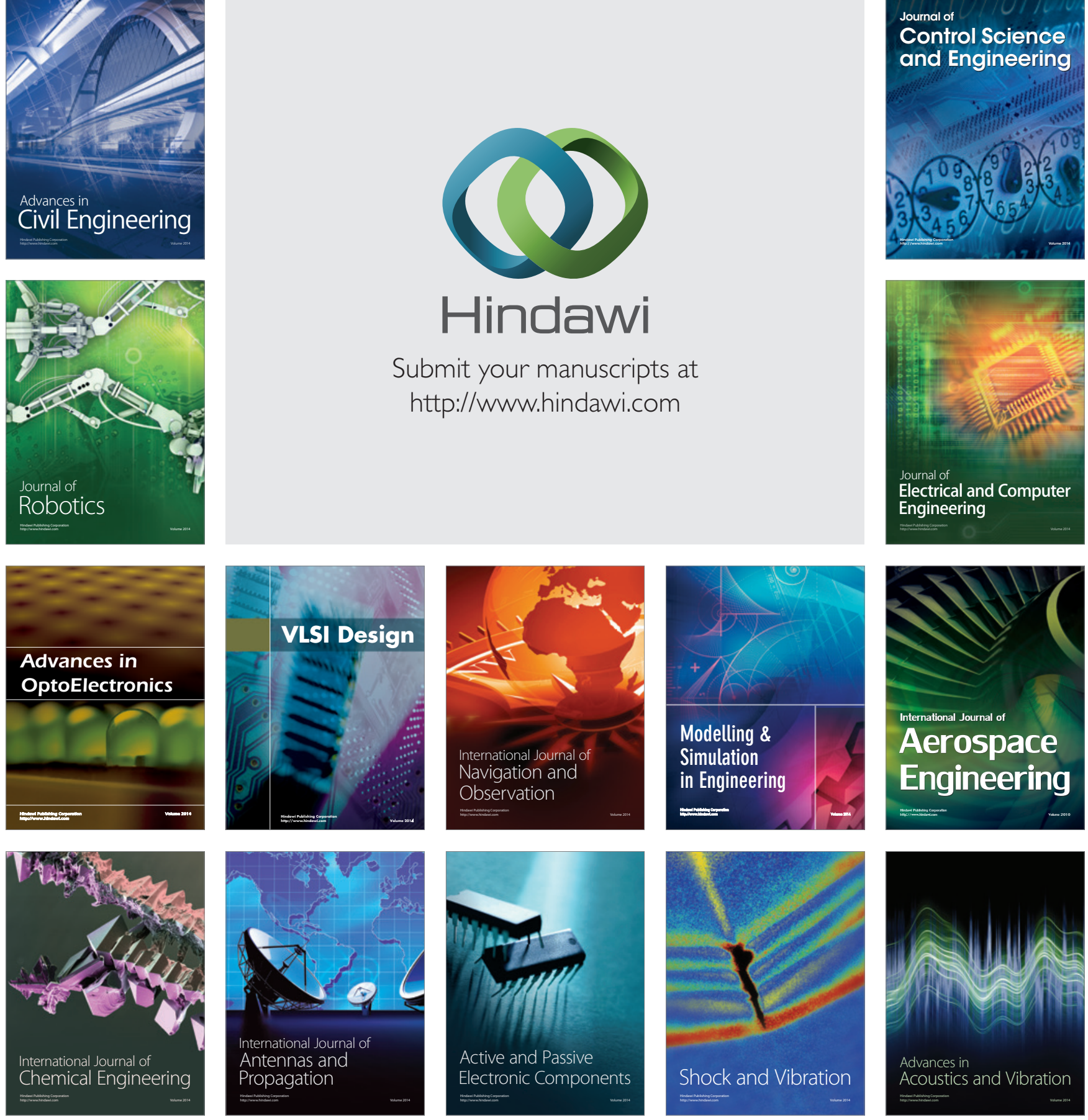